\title{
Pilot clinical study to assess caries lesion activity using quantitative light- induced fluorescence during dehydration
}

Masatoshi Ando

Andrea G. Ferreira-Zandoná

George J. Eckert

Domenick T. Zero

George K. Stookey 


\title{
Pilot clinical study to assess caries lesion activity using quantitative light-induced fluorescence during dehydration
}

\author{
Masatoshi Ando, ${ }^{a, \star}$ Andrea G. Ferreira-Zandoná, ${ }^{b}$ George J. Eckert, ${ }^{c}$ Domenick T. Zero, ${ }^{a}$ and \\ George K. Stookey ${ }^{d}$ \\ aIndiana University School of Dentistry, Department of Cariology, Operative Dentistry and Dental Public Health, Indianapolis, Indiana, \\ United States \\ bUniversity of North Carolina at Chapel Hill, School of Dentistry Department of Operative Dentistry, Chapel Hill, North Carolina, United States \\ 'Indiana University School of Medicine, Department of Biostatistics, Indianapolis, Indiana, United States \\ ${ }^{\mathrm{d} T h e r a m e t r i c ~ T e c h n o l o g i e s, ~ I n c ., ~ N o b l e s v i l l e, ~ I n d i a n a, ~ U n i t e d ~ S t a t e s ~}$
}

\begin{abstract}
This study aimed to evaluate the ability of quantitative light-induced fluorescence (QLF) to assess caries lesion activity using visual examination (VE) as the gold standard. Twenty-four visible white spot lesions on buccal surfaces were examined from 23 children, ages 9 to 14 years. At baseline, the surface was hydrated with water, and thereafter, it was dehydrated with continuous compressed air during image acquisition. QLF images were acquired at 0 (baseline), 5, and $15 \mathrm{~s}$. QLF variables [QLFV: fluorescence loss $(\Delta F)$, lesion size (S), $\Delta \mathrm{Q}: \Delta \mathrm{F} \times \mathrm{S}$ ] was recorded. Changes-in-QLF $F_{\mathrm{V}}$ per second $\left(\Delta \mathrm{QLF}_{\mathrm{V}}\right)$ were determined: $\Delta \mathrm{Q} \mathrm{F}_{\mathrm{V}}=$ $\left(\mathrm{QLF}_{\mathrm{VN}}-\mathrm{QLF}_{\mathrm{VBaseline}}\right) / N$, where $N$ indicates dehydration time. One experienced dentist conducted VE independently using a dental unit's light, compressed air, and explorer. QLF $F_{\mathrm{V}}$ and $\triangle Q L F_{\mathrm{V}}$ of the active group $(n=11)$ were compared with those of the inactive group $(n=13)$ using two-sample $t$-tests. As the surface was dehydrated, $S$ and $\Delta Q$ values of the active group increased, whereas QLF $F_{V}$ of the inactive group showed only a small change. $\triangle Q L F_{V}$ of the active group were larger than those of the inactive group; however, the difference did not reach statistical significance $(p>0.11)$. Within the limitations of this study, QLF data indicated increments for lesions designated as active and minimal change for lesions defined as inactive. ( 2017 Society of Photo-Optical Instrumentation Engineers (SPIE) [DOI: 10.1117/1.JBO.22.3.035005]
\end{abstract}

Keywords: clinical study; caries lesion activity; diagnostics; fluorescence.

Paper 160859R received Dec. 15, 2016; accepted for publication Feb. 21, 2017; published online Mar. 10, 2017.

\section{Introduction}

Dental caries is a dynamic process during which demineralization and remineralization cycles take place continuously. When the demineralization process continues, it increases surface porosity and creates white spot lesions due to increasing refractive index. ${ }^{1,2}$ Leaving these lesions untreated can lead to cavitation. By detecting the early stage of dental caries, such as white spot lesions, it allows to arrest the demineralization process before surgical treatment is considered and even to gain net mineral. In order to achieve this, early detection and caries activity assessment are critical. In addition to visual methods, several technology-based methods have been introduced.

Quantitative laser- and light-induced fluorescence (QLF) techniques are among the most widely studied methods for detection of early caries. They have been used widely in various in vitro, in situ, and in vivo studies. ${ }^{3-6}$ A study showed both laser and light fluorescence to be useful in detecting and quantifying white spot lesions. ${ }^{4}$ QLF has been used in a controlled clinical trial to show significant differences between fluoride dentifrice treatment regimens in as little as 3 months. ${ }^{6}$ This suggests that the use of QLF in clinical trials may reduce the time necessary to evaluate new therapeutic agents, saving both time and expense. The main advantage of this technique is that it can be used to quantify changes in carious lesions over time. ${ }^{5}$
Near-infrared (NIR) imaging has been shown to detect and quantify dental caries. ${ }^{7,8}$ Also, both thermal and NIR reflectance imaging have been found to be suitable for the detection of remineralization of simulated caries lesions. ${ }^{9}$ The use of optical coherence tomography (OCT) for caries detection and quantification has also shown promising results. ${ }^{10,11}$ Another study reported that polarization-sensitive OCT (PS-OCT) and dehydration rate measurements with NIR reflectance and thermal imaging methods were ideally suited for nondestructive, noninvasive, and quantitative measurement of lesion activity. ${ }^{12}$

Based on chemical analysis and histopathological observations, the initial stage of caries development is characterized by the opening of the intercrystalline spaces without the destruction of the surface and subsequent creation of microchannels. ${ }^{13-}$ ${ }^{15}$ These microchannels are found to be about 0.5 to $1.5 \mu \mathrm{m}$ in width in artificial lesions ${ }^{5}$ and range from 0.2 to $1.0 \mu \mathrm{m}$ in width in early natural enamel lesions. ${ }^{16}$ When white spot lesions (the early stage of demineralized enamel) are hydrated/wet, microchannels in lesions are filled with fluid/water. The presence or absence of water in microchannels affects the amount of light scatter and internal reflection. The difference between the refractive indices of water (1.33) and enamel crystal (1.62) is minimal. Because this difference is small, when the surface is wet (hydrated), there is less scatter and the light path is longer than 
when the surface is dry (dehydrated). Light absorption and fluorescence remitted per volume is much greater in the hydrated white spot lesions and fluorescence is stronger. This may be the reason white spot lesions may not be readily noticeable when the surface is wet (hydrated). However, when the lesions are dehydrated/dry, air fills the porous areas (intercrystalline spaces). Air has a lower refractive index (nearly 1.0). Differences between the refractive indices of intercrystalline space (air) and enamel crystal increase, hence the amount of light scattering increases. Light absorption and fluorescence remitted per volume is much smaller in the dehydrated white spot lesions and fluorescence is weaker. This may be the reason the white spot lesions are more readily recognized when the surface is dry (dehydrated).

A caries lesion that is progressing (continuing to demineralize) is described as an active caries lesion. A lesion that has stopped further progression (stagnant/remineralized) is referred to as an inactive or arrested caries lesion. ${ }^{17}$ There are several reports on the characteristics of caries lesion activity in the literature. ${ }^{18-20} \mathrm{~A}$ high reliability for assessment of caries lesion activity was shown with criteria based on observations of surface appearance and texture. ${ }^{21,22}$ For intact surfaces, active caries lesions were described as presenting a whitish/yellowish opaque enamel surface with a loss of luster and a rough texture when the tip of the probe is moved gently across the surface, whereas inactive caries lesions were described as presenting a shiny enamel surface and a hard and smooth texture. ${ }^{21}$ By definition, active caries lesions must have microchannels (increased porosity) that allow biofilm-generated acids to penetrate into the lesion body for caries to progress. On the other hand, inactive lesions should have smaller and/or fewer microchannels, indicating the caries process has been reversed or arrested. When lesions are wet, microchannels in active white spot lesions are filled with water and the amount of water is larger than in inactive white spot lesions. Inactive white spot lesions have smaller microchannels relative to active lesions, therefore, they have less water/fluid. As the size and/or number of microchannels increases, the surface porosity increases, thus water/fluid inside the intercrystalline spaces evaporates faster. Therefore, the central hypothesis of this study was that the rate and amount of vaporization (evaporation) of fluid in the lesion body during dehydration as measured by QLF indicates caries lesion activity. The change-in-QLF variables per second $\left(\Delta \mathrm{QLF}_{\mathrm{V}}\right)$ during dehydration would show relatively large values due to the presence of wider/longer microchannels and larger amounts of water in the lesion.

Ando et al. ${ }^{23}$ demonstrated in extracted human teeth that during the first few seconds of dehydration by continuous compressed air, change-in-QLF variables per second $\left(\Delta \mathrm{QLF}_{\mathrm{V}}\right)$ values for active lesions were larger than those of inactive lesions. This suggests that $\Delta \mathrm{QLF}_{\mathrm{V}}$ during the first few seconds of dehydration by continuous compressed air may be able to differentiate between active (demineralizing) and inactive (remineralized) caries lesions at the time of examination. To the authors' best knowledge, QLF during dehydration had not been tested clinically to assess caries lesion activity. Therefore, the objective of this clinical pilot study was to determine if changes-in-QLF variables during dehydration could be used to assess caries lesion activity of noncavitated caries lesions (white spot lesions) on the buccal surface in permanent teeth of children in vivo. Accomplishing the specific objective of this pilot clinical study would be of substantial significance for dental public health. Particularly, this study would evaluate the use of the objective and quantitative QLF during dehydration technique for determination of caries lesion activity at the time of examination. Using an objective and quantitative method (QLF with dehydration) could potentially improve clinical decision-making for more effective caries management.

\section{Subjects and Methods}

This study protocol was approved by the Indiana University Institutional Review Board (IRB \#0105-10). The subject's parents completed an informed consent medical/dental history and children signed an assent. Upon review and subsequent acceptance into the study, a visual examination (VE) was performed on all of the teeth in the mouth. Subjects who had at least one white spot lesion, no severe cavitated caries lesions, no moderate to severe periodontal diseases, and no orthodontic appliances were included. Twenty-three children, ages 9 to 14 years, presented 24 visible white spot lesions on the buccal surface of permanent teeth at the gingival margin for examination. One experienced dentist, trained and calibrated in the International Caries Detection and Assessment System $(\text { ICDAS })^{24}$ and Nyvad's criteria, ${ }^{21}$ independently conducted the VE to assess caries lesion activity using a dental unit's light and compressed air, with an explorer used only to check surface structure. The examiner utilized the best knowledge from both criteria to assess caries lesion activity using reflection and tactile sensation. Active caries lesions were defined as presenting a whitish/yellowish opaque enamel surface with a loss of luster and a rough texture when the tip of the explorer was moved gently across the surface. Inactive caries lesions were defined as presenting a shiny enamel surface and a hard and smooth texture when the tip of the explorer was moved gently across the surface. In order to reduce/eliminate bias, a separate examiner performed an examination using QLF with the dehydration technique.

\subsection{Quantitative Light-Induced Fluorescence Examination}

Three consecutive fluorescence images per surface were acquired at 0 (baseline), 5 , and $15 \mathrm{~s}$ with the quantitative lightinduced fluorescence system (QLFPatient 3.0.0.35, Inspektor Research Systems B.V., The Netherlands). These intervals between image acquisition were based on the previous in vitro study. ${ }^{23}$ The state of hydration of the surfaces was standardized by placing a wet cotton pellet, which was soaked in distilled water, on the buccal surface for $60 \mathrm{~s}$. The QLF camera handpiece was maintained in a position that provided the best illumination of the buccal surface. The subject bit and held a short piece of wooden tongue depressor and the QLF camera handpiece was placed against this to secure the position. During image acquisition while the examiner maintained the QLF camera position, the lesions were dehydrated with continuous compressed air by an assistant. After the image acquisition, QLF variables $\left(\mathrm{QLF}_{\mathrm{V}}\right)$ of fluorescence loss $[\Delta \mathrm{F}(\%)]$, lesion size [S $\left.\left(\mathrm{mm}^{2}\right)\right]$, and $\Delta \mathrm{Q}: \Delta \mathrm{F} \times \mathrm{S}\left(\% \times \mathrm{mm}^{2}\right)$ were determined with proprietary QLF software (QLF $2.00 \mathrm{~g}$, Inspektor Research Systems B.V., The Netherlands). The details of image analysis with QLF software were described previously. ${ }^{10}$ Briefly, a lesion threshold $<95 \%$ of reconstructed sound fluorescence radiance level was considered to be a caries lesion. 


\subsection{Data Analyses}

Changes-in-QLF variables per second $\left[\Delta \mathrm{QLF}_{\mathrm{VN}}: \Delta \mathrm{F}_{\mathrm{VN}}(\% / \mathrm{s})\right.$, $\left.\Delta \mathrm{S}_{\mathrm{VN}}\left(\mathrm{mm}^{2} / \mathrm{s}\right), \Delta \mathrm{Q}_{\mathrm{VN}}\left(\% \times \mathrm{mm}^{2} / \mathrm{s}\right)\right]$ were determined using

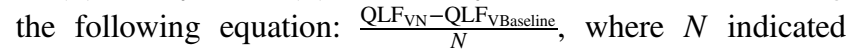
dehydration/image acquisition time.

$\mathrm{QLF}_{\mathrm{V}}$ and $\Delta \mathrm{QLF}_{\mathrm{V}}$ values were divided into active and inactive white spot lesion groups based on the VE. The averages and standard errors of $\mathrm{QLF}_{\mathrm{V}}$ and $\Delta \mathrm{QLF}_{\mathrm{V}}$ values of each dehydration (image acquisition) time were calculated for the active and inactive groups. Comparisons between active and inactive white spot lesions at each dehydration time were performed using 2-sample $t$-tests, and comparisons of the measurements during dehydration were made using repeated measures ANOVA. A 5\% significance level was used for all tests. Statistical analyses were performed using SAS version 9.1 (SAS Institute Inc., Cary, North Carolina).

\section{Results}

Eleven out of 24 sites were designated as active status according to VE, and 13 sites were designated as inactive status. Figure 1 shows examples of fluorescence images of both active and inactive groups at 0,5 , and $15 \mathrm{~s}$ of dehydration. Caries lesions appeared darker than sound area. As dehydration time increased, active caries lesion got darker. On the other hand, inactive caries lesion did not get darker. Figure 2 displays the average and standard error of fluorescence loss. Although there were no statistically significant differences between groups ( $0 \mathrm{~s}: p=0.16$, $5 \mathrm{~s}: p=0.09,15 \mathrm{~s}: p=0.14$ ) or among dehydration times (active: $0 \mathrm{~s}$ versus $5 \mathrm{~s}: p=0.92,0 \mathrm{~s}$ versus $15 \mathrm{~s}: p=0.59$, $5 \mathrm{~s}$ versus $15 \mathrm{~s}: p=0.53$; inactive: $0 \mathrm{~s}$ versus $5 \mathrm{~s}: p=0.44$,

\begin{tabular}{|c|c|c|c|}
\hline \multirow{2}{*}{$\begin{array}{c}\text { Activity } \\
\text { status }\end{array}$} & \multicolumn{3}{|c|}{ Dehydration / image acquisition time (s) } \\
\cline { 2 - 4 } Active & 0 (Baseline) & 5 & 15 \\
\hline \multirow{2}{*}{ Inactive } & & & \\
\hline
\end{tabular}

Fig. 1 Example of fluorescence images of both active and inactive groups at 0 (baseline), 5 , and $15 \mathrm{~s}$ of dehydration.

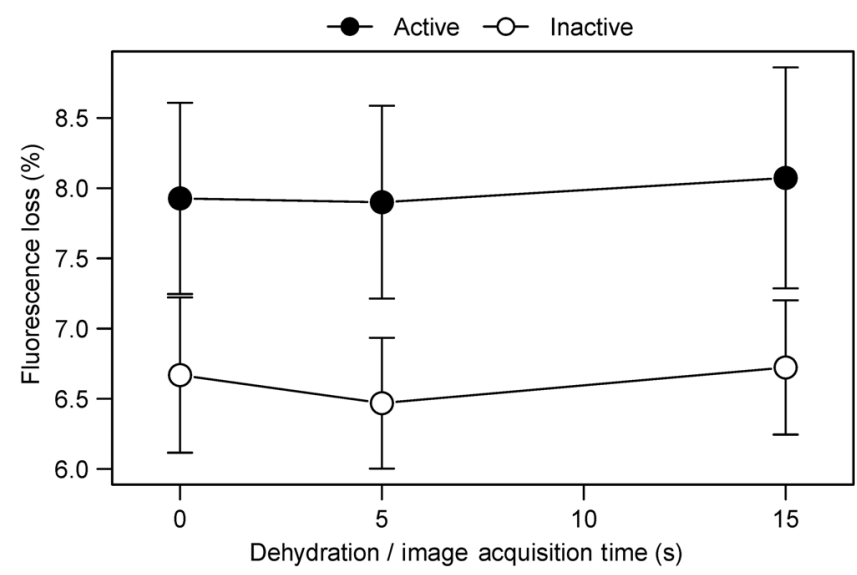

Fig. 2 Average and standard error of fluorescence loss for active and inactive groups. There were no significant differences between groups or among dehydration times $(p>0.05)$.

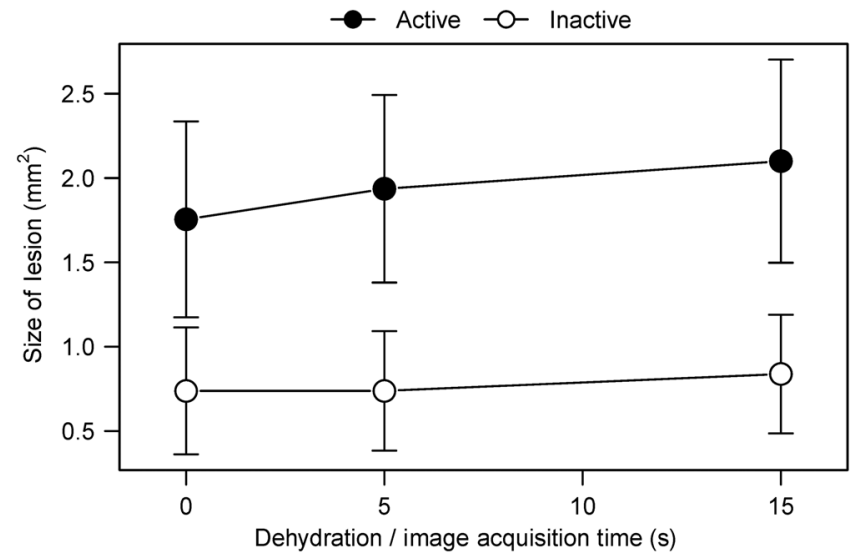

Fig. 3 Average and standard error of size of lesion for active and inactive groups. There were no significant differences between groups or among dehydration times $(p>0.05)$.

$0 \mathrm{~s}$ versus $15 \mathrm{~s}: p=0.82,5 \mathrm{~s}$ versus $15 \mathrm{~s}: p=0.35)$, more loss of fluorescence was observed in the active lesions. However, dehydration for either 5 or $15 \mathrm{~s}$ did not alter the amount of fluorescence loss for either type of lesion.

Figure 3 similarly presents the effect of dehydration on the size of the lesions as assessed by QLF. While again there were no statistically significant differences between groups $(0 \mathrm{~s}$ : $p=0.14,5 \mathrm{~s}: p=0.07,15 \mathrm{~s}: p=0.07)$ or among dehydration times (active: $0 \mathrm{~s}$ versus $5 \mathrm{~s}: p=0.22$, 0 s versus $15 \mathrm{~s}: p=0.08$, $5 \mathrm{~s}$ versus $15 \mathrm{~s}: p=0.25$; inactive: $0 \mathrm{~s}$ versus $5 \mathrm{~s}: p=1.00,0 \mathrm{~s}$ versus $15 \mathrm{~s}: p=0.40,5 \mathrm{~s}$ versus $15 \mathrm{~s}: p=0.40)$, active lesions were observed to be larger in size $\left(\mathrm{mm}^{2}\right)$. Dehydration did not have obvious influence on apparent lesion size for the inactive lesions, but an apparent increase in lesion size was observed for active lesions following dehydration.

Figure 4 shows the impact of dehydration on the volume $(\Delta \mathrm{Q})$ of active and inactive lesions. There were no statistically significant differences between groups ( $0 \mathrm{~s}: p=0.22,5 \mathrm{~s}$ : $p=0.11,15 \mathrm{~s}: p=0.12$ ) or among dehydration times (active: $0 \mathrm{~s}$ versus $5 \mathrm{~s}: p=0.41,0 \mathrm{~s}$ versus $15 \mathrm{~s}: p=0.11,5 \mathrm{~s}$ versus $15 \mathrm{~s}: p=0.22$; inactive: $0 \mathrm{~s}$ versus $5 \mathrm{~s}: p=0.57,0 \mathrm{~s}$ versus $15 \mathrm{~s}: p=1.00,5 \mathrm{~s}$ versus $15 \mathrm{~s}: p=0.56)$. Influenced by the results for lesion size, dehydration had little effect on the volume $(\Delta \mathrm{Q})$ of inactive lesions but was increased slightly in active

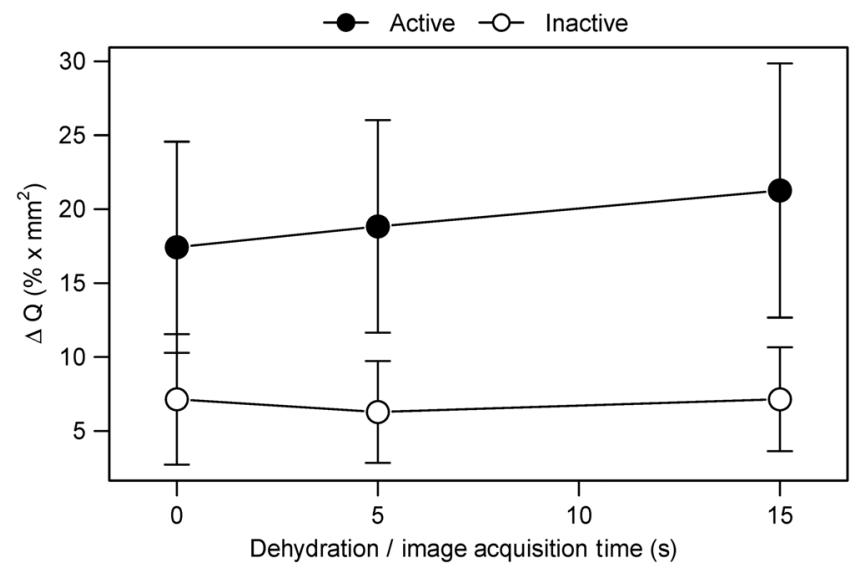

Fig. 4 Average and standard error of $\Delta Q$ for active and inactive groups. There were no significant differences between groups or among dehydration times $(p>0.05)$. 
Table 1 Means \pm standard error of the mean for change-in-QLF variables per second for active and inactive groups. The differences between groups were not statistically significant $(p>0.05)$.

\begin{tabular}{|c|c|c|c|c|c|c|}
\hline \multirow[b]{2}{*}{ Dehydration time (s) } & \multicolumn{2}{|c|}{$\Delta \mathrm{F}_{\mathrm{V}}(\% / \mathrm{s})$} & \multicolumn{2}{|c|}{$\Delta S_{V}\left(\mathrm{~mm}^{2} / \mathrm{s}\right)$} & \multicolumn{2}{|c|}{$\Delta Q_{V}\left(\% \times \mathrm{mm}^{2} / \mathrm{s}\right)$} \\
\hline & Active & Inactive & Active & Inactive & Active & Inactive \\
\hline 5 & $-0.005 \pm 0.034$ & $-0.040 \pm 0.034$ & $0.036 \pm 0.019$ & $0.000 \pm 0.010$ & $0.281 \pm 0.163$ & $-0.171 \pm 0.215$ \\
\hline 15 & $0.010 \pm 0.019$ & $0.004 \pm 0.012$ & $0.023 \pm 0.009$ & $0.007 \pm 0.004$ & $0.256 \pm 0.137$ & $0.000 \pm 0.066$ \\
\hline
\end{tabular}

lesions. This was in contrast to the $\mathrm{QLF}_{\mathrm{D}}$ of inactive lesions, which showed minimal change during dehydration.

Table 1 presents the average and standard error of the changes-in-QLF variables per second $\left(\Delta \mathrm{QLF}_{\mathrm{V}}\right)$ for the active and inactive groups. Although $\Delta \mathrm{QLF}_{\mathrm{V}}$ values of active lesions were larger than those of inactive lesions, the differences between groups did not reach statistical significance $\left(5 \mathrm{~s} \Delta \mathrm{F}_{\mathrm{V}}\right.$ : $p=0.49,5$ s $\Delta \mathrm{S}_{\mathrm{V}}: p=0.11,5 \mathrm{~s} \Delta \mathrm{Q}_{\mathrm{V}}: p=0.12,15 \mathrm{~s} \Delta \mathrm{F}_{\mathrm{V}}$ : $\left.p=0.78,15 \mathrm{~s} \Delta \mathrm{S}_{\mathrm{V}}: p=0.11,15 \mathrm{~s} \Delta \mathrm{Q}_{\mathrm{V}}: p=0.12\right)$.

To explore how the measurements fared as a diagnostic test, a cut-off value for the change-in- $\Delta \mathrm{Q}$ per second $\left(\Delta \mathrm{Q}_{\mathrm{V}}\right)$ was selected at $\Delta \mathrm{Q}_{\mathrm{V}}=1.2$. With this cut-off, sensitivity was $100 \%$, specificity was $62 \%$, positive predictive value was $69 \%$, and negative predictive value was $100 \%$. Given the small sample size in this study, this may not be the optimal cut-off; however, it does show that the method has relevance as a possible method to identify activity.

\section{Discussion}

In the postfluoride era, progression of enamel caries is slower, and it takes longer before the stage of irreversible destruction of the tooth is reached (cavitation). ${ }^{25}$ With new assessment methods, such as QLF, caries detection is possible at an earlier stage than could be performed previously. This is very important for carrying out timely and appropriate preventive measures. However, as caries is an arrestable and reversible process in its early stages, identification of only the severity of caries lesions, such as lesion size and depth, is insufficient. We know some of these lesions will be active and, therefore, in need of some form of intervention, while others may be a scar of past damage (inactive/arrested) and, therefore, will not require any intervention. In order to select an appropriate treatment modality and aid in the clinical decision-making process, caries diagnosis and assessment of caries lesion activity are of critical importance. Incorrect diagnosis can easily result in incorrect treatment decisions, particularly with respect to irreversible treatments (i.e., restorations).

The key feature that allows QLF imaging to differentiate between active and inactive lesions is based on their differences in the surface structure, specifically the porosity of the surface layer. As mentioned previously, active lesions may present a porous surface layer and inactive lesions may have a relatively nonporous surface layer. The porosity depends on the number and size of microchannels. A study using scanning electron microscopy indicated that microchannels developed at caries initiation and increased in size with continued demineralization. ${ }^{26}$ As the size and/or number of microchannels increases, the surface porosity increases. On the other hand, inactive lesions should have fewer and narrower microchannels. This implies that the surface porosity decreases. For active caries lesions, based on our hypothesis, the $\mathrm{QLF}_{\mathrm{V}}$ and $\Delta \mathrm{QLF} \mathrm{V}_{\mathrm{V}}$ during dehydration would show relatively large values, due to the presence of wider/longer microchannels, and larger amounts of water in the lesion. On the other hand, for inactive caries lesions, $\mathrm{QLF}_{\mathrm{V}}$ and $\Delta \mathrm{QLF}_{\mathrm{V}}$ would show much lower values during dehydration, due to the presence of smaller and fewer microchannels, and thus a negligible amount of water in the lesion.

In this pilot clinical study, although not reaching statistical significance, the results appear to support our hypothesis by indicating there was a trend for $\Delta \mathrm{Q}$ of active lesions to increase as the surface was dehydrated, leading to higher change-in- $\Delta \mathrm{Q}$ $\left(\Delta \mathrm{Q}_{\mathrm{V}}\right)$ for active lesions than for inactive lesions. A poststudy power analysis was performed to evaluate the role of the small sample size on the lack of statistical significance for this study. The observed differences in $\Delta \mathrm{Q}_{\mathrm{V}}$ were 0.45 and 0.25 at 5 and $15 \mathrm{~s}$ of dehydration, respectively. A study with $n=42$ per group would have $80 \%$ power at a $5 \%$ significance level to detect the $\Delta \mathrm{Q}_{\mathrm{V}}$ differences we observed. Therefore, this pilot clinical study suggests that QLF imaging of caries lesions undergoing dehydration can be used to differentiate caries lesion activity. This is advantageous as it can be done at the time of examination, and information can be obtained in a single appointment. However, further investigations are needed to determine the $\Delta \mathrm{Q}_{\mathrm{V}}$ threshold to differentiate active and inactive caries lesions.

There were two other studies of fluorescence imaging with dehydration that may be relevant to this study. Al-Khateeb et al. ${ }^{27}$ indicated that dehydration data could serve as a separate method for analyzing pore distribution properties in lesions to differentiate history of deremineralization or fluoride treatment. van der Veen et al. ${ }^{28}$ demonstrated the potential to use fluorescence imaging with dehydration to determine caries lesion activity. For both studies, dehydration was done by allowing the specimens to dry at room temperature rather than using continuous compressed air. These studies may not be directly applicable to a clinical setting. Clinically another study has demonstrated that lesions which progress to cavitation (active) present faster changes in $\mathrm{QLF}_{\mathrm{V}}$ than inactive lesions. ${ }^{5}$ Another clinical study, using a different laser fluorescence device (LF: this consists of a 655-nm emitted diode laser) with occlusal caries, demonstrated that after air drying for 3 and $15 \mathrm{~s}$, LF values of active caries, in which caries activity was determined by VE, presented higher than those of inactive caries. ${ }^{29}$

There were also studies regarding the dehydration effect using OCT. For one study, dehydration was induced by the specimens in air at room temperature for $30 \mathrm{~min}$. The results showed that hydration state affects the reflectivity of demineralized porous enamel, and the effect can be potentially used for assessment of early enamel lesion using OCT. ${ }^{30}$ For another study, dehydration was produced in a controlled environment for $30 \mathrm{~s}$. The air pressure was set to $15 \mathrm{psi}$, and the computer-controlled air nozzle was positioned $2 \mathrm{~cm}$ away from the sample. ${ }^{31}$ Although bovine dentin and extracted human root caries were used, 
thermal imaging and PS-OCT may be ideally suited for the nondestructive root caries lesion activity during a clinical examination. Therefore, these studies and this study suggested that the state of hydration in the lesion body can be used to quantify severity of dental caries and caries lesion activity.

For this proof-of-concept study, we intended to demonstrate that QLF data could indicate increments for lesions designated as active and minimal change for lesions defined as inactive. Hence, the design of this study was small sample size and cross sectional proof-of-concept pilot study. VE was employed as the gold standard. The examiner for VE in this study is trained and calibrated with ICDAS ${ }^{24}$ and Nyvad's criteria ${ }^{21}$ and is expert in caries detection and diagnosis. Previous studies demonstrated that both criteria could predict lesion depth and assess caries lesion activity. ${ }^{21,22,24,32-34}$ Although not reaching statistical significance, this small, pilot clinical study provided preliminary information to establish the use of the QLF method. Further clinical investigations to establish threshold or cut-off values to distinguish among the lesion stages and a larger sample size for clinical application will be necessary.

\section{Conclusion}

The results of this small, pilot clinical study suggest that QLF data, especially $\Delta \mathrm{Q}$, indicated slight increments for lesions designated as active and minute changes for lesions defined as inactive. Furthermore, change-in- $\Delta \mathrm{Q}$ per second $\left(\Delta \mathrm{Q}_{\mathrm{V}}\right)$ of active lesions was greater than that of inactive lesions. Therefore, QLF during dehydration has the potential to assess caries lesion activity of noncavitated caries lesions (white spot lesions) on smooth surface in vivo at the time of examination.

\section{Disclosures}

George K. Stookey is the owner of Therametric Technologies, Inc.; he has an interest in the use of fluorescence for early caries detection, but no financial interest in QLF. The other authors declare no conflict of interest.

\section{Acknowledgments}

This study was supported by the 21 st Century Research and Technology Fund from the State of Indiana, USA. The funder had no role in study design, data collection and analysis, decision to publish, or preparation of the paper.

\section{References}

1. Z. Meng et al., "Measurement of the refractive index of human teeth by optical coherence tomography," J. Biomed. Opt. 14(3), 034010 (2009).

2. I. Hariri et al., "Estimation of the enamel and dentin mineral content from the refractive index," Caries Res. 47(1), 18-26 (2013).

3. S. Al-Khateeb et al., "Laser fluorescence quantification of remineralization in situ of incipient enamel lesions: influence of fluoride supplements," Caries Res. 31(2), 132-140 (1997).

4. M. Ando et al., "Comparative study to quantify demineralized enamel in deciduous and permanent teeth using laser- and light-induced fluorescence techniques," Caries Res. 35(6), 464-470 (2001).

5. A. F. Zandoná et al., "Longitudinal analyses of early lesions by fluorescence: an observational study," J. Dent. Res. 92(S1), S84-S89 (2013).

6. M. Kambara et al., "Results of clinical trial of fluoride dentifrices using QLF," in Early Detection of Dental Caries III, G. K. Stookey, Ed., pp. 229-235, Indiana University School of Dentistry, Indianapolis, Indiana (2003).

7. C. Zakian, I. Pretty, and R. Ellwood, "Near-infrared hyperspectral imaging of teeth for dental caries detection," J. Biomed. Opt. 14(6), 064047 (2009).
8. S. Salsone et al., "Histological validation of near-infrared reflectance multispectral imaging technique for caries detection and quantification," J. Biomed. Opt. 17(7), 076009 (2012).

9. R. C. Lee, C. L. Darling, and D. Fried, "Assessment of remineralization via measurement of dehydration rates with thermal and near-IR reflectance imaging," J. Dent. 43(8), 1032-1042 (2015).

10. T. Ueno et al., "Optical analysis of enamel and dentin caries in relation to mineral density using swept-source optical coherence tomography," J. Med. Imaging 3(3), 035507 (2016).

11. A. M. Maja et al., "Evaluation of dental enamel caries assessment using quantitative light induced fluorescence and optical coherence tomography," J. Biophotonics 9(6), 596-602 (2016).

12. R. C. Lee et al., "Infrared methods for assessment of the activity of natural enamel caries lesions," IEEE J. Sel. Top. Quantum Electron. 22(3), 6803609 (2016).

13. M. Goldberg et al., "Microchannels in the surface zone of artificially produced caries-like enamel lesions," J. Biol. Buccale 9(3), 297-314 (1981).

14. J. D. B. Featherstone et al., "Chemical and histological changes during development of artificial caries," Caries Res. 19(1), 1-10 (1985).

15. L. Holmen et al., "A scanning electron microscopy study of surface changes during development of artificial caries," Caries Res. 19(1), 11-21 (1985).

16. R. M. Frank and A. Brendel, "Ultrastructure of the approximal dental plaque and the underlying normal and carious enamel," Arch. Oral Biol. 11(9), 883-912 (1966).

17. O. Fejerskov, B. Nyvad, and E. A. M. Kidd, "Clinical and histological manifestations of dental caries," in Dental Caries The Disease and its Clinical Management, O. Fejerskov and E. A. M. Kidd, Eds., pp. 71-98, Blackwell, Munksgaard, Oxford (2003).

18. L. Holmen, A. Thylstrup, and J. Årtun, "Surface changes during the arrest of active enamel carious lesions in vivo: a scanning electron microscopy," Acta Odontologica Scandinavica 45(6), 383-390 (1984).

19. A. Thylstrup, C. Bruun, and L. Holman, "In vivo caries models: mechanisms for caries initiation and arrestment," Adv. Dent. Res. 8(2), 144157 (1994).

20. B. ten Cate, "The role of saliva in mineral equilibria-caries, erosion and calculus formation," in Saliva and Oral Health, 3rd ed., M. Edgar, C. Dawes, and D. O'Mullane, Eds., pp. 120-135, British Dental Association, London (2004).

21. B. Nyvad, V. Machiulskiene, and V. Baelum, "Reliability of a new caries diagnostic system differentiating between active and inactive caries lesions," Caries Res. 33(4), 252-260 (1999).

22. B. Nyvad, V. Machiulskiene, and V. Baelum, "Construct and predictive validity of clinical caries diagnostic criteria assessing lesion activity," J. Dent. Res. 82(2), 117-122 (2003).

23. M. Ando, G. K. Stookey, and D. T. Zero, "Ability of quantitative lightinduced fluorescence (QLF) to assess the activity of white-spot lesions during dehydration," Am. J. Dent. 19(1), 15-18 (2006).

24. A. I. Ismail et al., "Reliability of the international caries detection and assessment system (ICDAS): an integrated system for measuring dental caries," Community Dent. Oral Epidemiol. 35(3), 170-178 (2007).

25. E. Newbrun, "Preventing dental caries: current and prospective strategies," J. Am. Dent. Assoc. 123(5), 68-73 (1992).

26. M. Ando et al., "Characteristics of early stage of enamel demineralization in vitro," in Early Detection of Dental Caries III, G. K. Stookey, Eds., pp. 363-373, Indiana University School of Dentistry, Indianapolis, Indiana (2003).

27. S. Al-Khateeb et al., "Light-induced fluorescence studies on dehydration of incipient enamel lesions," Caries Res. 36(1), 25-30 (2002).

28. M. H. van der Veen, E. de Josselin de Jong, and S. Al-Khateeb, "Caries activity detection by dehydration with quantitative light fluorescence," in Early Detection of Dental Caries II, G. K. Stookey, Ed., pp. 251-259, Indiana University School of Dentistry, Indianapolis, Indiana (1999).

29. M. Braga et al., "New methodology to assess activity status of occlusal caries in primary teeth using laser fluorescence device," J. Biomed. Opt. 15(4), 047005 (2010).

30. A. Nazari et al., "Effect of hydration on assessment of early enamel lesion using swept-source optical coherence tomography," J. Biophotonics 6(2), 171-177 (2013).

31. R. C. Lee et al., "Activity assessment of root caries lesions with thermal and near-IR imaging methods," J. Biophotonics (2016). 
32. L. Shoaib et al., "Validity and reproducibility of ICDAS II in primary teeth," Caries Res. 43(6), 442-448 (2009).

33. M. C. Séllos and V. M. Soviero, "Reliability of the Nyvad criteria for caries assessment in primary teeth," Eur. J. Oral Sci. 119(3), 225-231 (2011).

34. R. S. Guedes et al., "Validation of visual caries activity assessment: a 2-yr cohort study," J. Dent. Res. 93(7 Suppl.), 101S-107S (2014).

Masatoshi Ando is an associate professor in the Department of Cariology, Operative Dentistry, and Dental Public Health at Indiana University School of Dentistry. His main focus is to develop an objective and quantitative means to measure caries lesion activity. He has extensive experience in the application of QLF and histological validation of lesions, using such techniques as microfocus computed tomography, transverse microradiography, optical reflecmetry, optical surface profilometry, and confocal laser scanning microscopy.
Andrea G. Ferreira-Zandoná is an associate professor of the Department of Operative Dentistry, University of North Carolina at Chapel Hill, School of Dentistry.

George J. Eckert is a biostatistician supervisor of the Department of Biostatistics, Indiana University School of Medicine.

Domenick T. Zero is a professor of the Department of Cariology, Operative Dentistry, and Dental Public Health at Indiana University School of Dentistry.

George K. Stookey is a chief executive officer and president of Therametric Technologies, Inc., and a distinguished professor emeritus at Indiana University. 